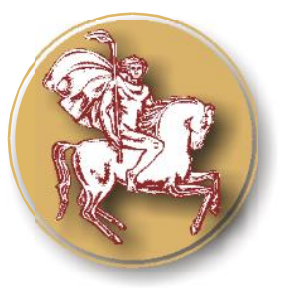

Trakia Journal of Sciences, No 3, pp 290-294, 2019

Copyright (C) 2019 Trakia University

Available online at:

ISSN 1313-7050 (print)

http://www.uni-sz.bg

ISSN 1313-3551 (online)

doi:10.15547/tjs.2019.03.019

Review

\title{
BIOMARKERS FOR UNSTABLE ATHEROSCLEROTIC PLAQUES IN CAROTID ARTERIES
}

\author{
M. Peycheva ${ }^{1 *}$, Z. Zahariev ${ }^{1}$, T. Deneva ${ }^{3}$ \\ ${ }^{1}$ Department of Neurology, Medical University- Plovdiv, Plovdiv, Bulgaria \\ ${ }^{2}$ Department of Clinical laboratory, Medical University- Plovdiv, Plovdiv, Bulgaria
}

\begin{abstract}
Carotid atherosclerosis is one of the main cerebro-vascular risk factors. It is a complex inflammatory disease resulting in lipid accumulation in the artery wall. The efforts are directed to find reliable biomarkers that can predict the atherosclerotic burden, plaque instability, plaque transformation and the risk for the ischaemic stoke. The modification of the inflammatory factors will help in finding new promising treatment options. Future treatment strategies are directed to stratified medicine - better risk assessment for each patient and optimization of personal management.
\end{abstract}

Key words: unstable carotid plaque, atherosclerosis, biomarkers, vascular risk

\section{INTODUCTION}

Atherosclerosis is a chronic and systemic disease characterized with the formation of atherosclerotic plaques, affecting mainly medium and large size arteries (1-3). Carotid atherosclerosis is a focal expression of the disease and is one of the leading cause for cerebro-vascular accidents (4).

The French pathologist Jean Lobstein first used the term "arteriosclerosis" (5). Lately Virchow suggests that immune mechanisms are involved in the processes of atherosclerosis. In the past this process was mainly characterized with lipid accumulation in the arterial wall. Nowadays it is accepted as a complex inflammatory disease (6).

\section{The pathological mechanisms of carotid atherosclerosis (Figure 1)}

Atherosclerosis is a slowly progressive process, occurring at predilection sites of the arterial walls with disturbed laminar flow (7, 8). Carotid atherosclerosis is mainly at the bifurcation of the common carotid artery and

\footnotetext{
*Correspondence to: Marieta Peycheva, Medical University Plovdiv, bul.Peshtersko shosse 66, 4000 Plovdiv,Bulgaria, Mobile 0898658408, Emailmpeitcheva@yahoo.com
}

into the initial part of internal and external carotid arteries (9-11). Endothelial dysfunction and structural alterations permit subendothelial accumulation of LDLs (low-density lipoproteins) $(12,13)$. A complex cascade of immune mediated mechanisms are involved in the formation of atherosclerotic plaque. The dysfunctional endothelial cells express adhesion molecules (ICAM-1, VCAM-1, and p-selectin) allowing the adherence of monocytes, T-lymphocytes and platelets to the endothelium. Lately they activate proinflammatory and pro-thrombotic factors (6, 14). Monocytes migrate into the endothelium mediated by monocyte chemo attractant protein-1(MCP-1). In response of macrophage colony-stimulating factor (M-CSF), they differentiate into macrophages. They are highly phagocytic modified lipids. By receptor-mediated phagocytosis, the accumulation of lipids within intimal cells leads to the formation of foam cells. This culminates in the appearance of fatty streaks in the arteries, altered intima and formation of a lipid core (15). The lipid necrotic core becomes separated from the arterial lumen by a fibrous cap (16). The cap is formed mainly by smooth muscle cells that migrate and proliferate from adventitia to intima. As the process goes the plaque became unstable with 
lipid and calcium deposits, new vessels, cellular debris and active immune cells. The fibrous cap is tinning and tearing. The connective tissue in the cap can be weakened and modified by the matrix metalloproteinases (MMPs) (14, 17). The new vessels from the adventitial vasa vasorum and the inflammation lead to intraplaque hemorrhages. The plaque's surface becomes vulnerable to rupture and reveals the necrotic core prone to formation of thrombi (18). All these processes are mediated by different pro-inflammatory and antiinflammatory cytokines and mediators.

Ischaemic stroke mechanisms of extracranial carotid atherosclerosis are artery-to-artery embolism, hypoperfusion and the combination of them.

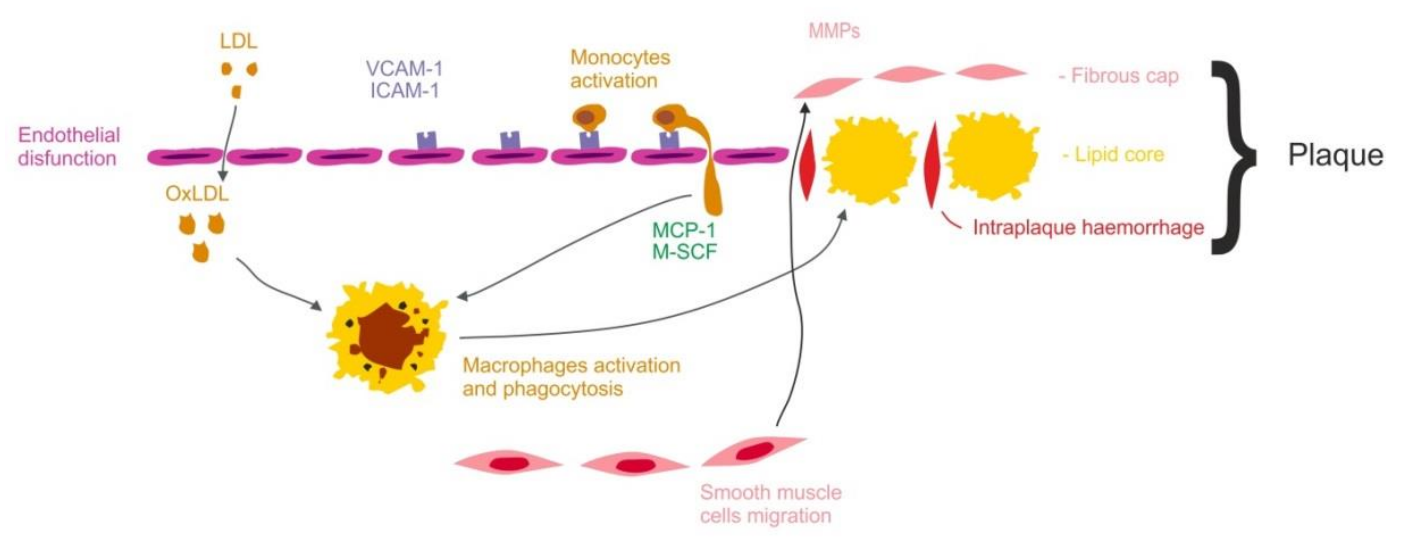

Figure 1. Pathological mechanism of atherosclerosis.

\section{Carotid plaque biomarkers (Table 1)}

Table 1. Biomarkers and their role in atherosclerosis

\begin{tabular}{|l|l|}
\hline Biomarkers & Role in atherosclerosis \\
\hline low-density lipoproteins & Formation of plaque lipid core \\
\hline acute phase protein (hs-CRP, PTX3) & $\begin{array}{l}\text { predictors for the presence of carotid plaque and } \\
\text { role in plaque activity }\end{array}$ \\
\hline cell adhesion molecules (ICAM-1 and VCAM-1) & $\begin{array}{l}\text { mediate leukocyte migration toward vascular } \\
\text { wall tissue and subendothelial adhesion }\end{array}$ \\
\hline Matrix Metalloproteinases & $\begin{array}{l}\text { role in matrix degradation and plaque } \\
\text { destabilization }\end{array}$ \\
\hline Fibrinogen & role in the coagulation cascade and thrombosis \\
\hline proinflammatory cytokines( Il-6,Il-18,Il-23,Il-27) & Plaque destabilization and instability \\
\hline
\end{tabular}

One of the most important risk factors for atherosclerosis is the plasma level of lowdensity lipoprotein cholesterol. In 2016 European Society of Cardiology and European Atherosclerosis Society published the new guidelines for the management of dyslipidemia (19). The Task force created the SCORE chart to estimate the 10 years fatal cerebrovascular risk and the treatment targets. The increased level of LDL-C, triglyceride-rich lipoproteins and some lipoprotein subtractions influence also vascular inflammation. They produce pro-inflammatory mediators that activate the mononuclear cells, disable the endothelial cells and impair functionally HDLC $(17,20,21)$.

Several investigations study the inflammatory mechanisms of atherosclerosis. The identification of proper immunological biomarker and its correlation with the cerebrovascular risk in patients with carotid atherosclerosis will give better diagnostic and treatment options. It is known that there are circulating biomarkers that reliably predict the risk of ischaemic stroke.

Large epidemiological studies as Physicians' Health Study (22), Women's Health Study (23), Framingam Heart Study task (24) find 
that high sensitive C-reactive protein ( hs-CRP ) in serum has a predictive role for ischaemic stroke. Studies indicate that the high serum level of hs-CRP can suppose the presence of atheroslerotic plaque in carotid arteries but without a correlation with the degree of the stenosis $(25,26)$. Studies on various inflammatory factors and the onset of a new cerebro-vascular accident, again find a connection with the high serum hs-CRP (24). It is also an independent predictor of the early carotid atherosclerosis as it participates in plaque activation (27).

Other acute phase protein that is connected with atherosclerosis is pentraxin-3(PTX3). Some data show that its plasma levels correlate with the presence of plaques (28). Immunohistochemical study of paraffin and frozen sections indicate that macrophages, mainly foam cells and neutrophils, expressed PTX3 in advanced atherosclerotic lesions (29). But its role as a predictor of cerebro-vascular accidents is still not investigated.

Of interest are several types of immunoglobulin-like cell adhesion molecules (CAM), whose function is to support, organize and mediate leukocyte migration toward vascular wall tissue, with subsequent stable adhesion and migration therein. Representatives of this group are the biomarkers ICAM -1 and VCAM-1. They have a proven role in the endothelial dysfunction and in the processes of atherosclerosis. VCAM-1 promotes adhesion of lymphocytes and monocytes by binding beta 1 integrins (30). ICAM -1 is a major endothelial ligand for LFA-1, which mediates strong adhesion of cells to damaged endothelium at the site of inflammation (31). The expression of ICAM -1 and VCAM-1 on the endothelial cells is after the stimulation of pro-inflammatory mediators. After the adhesion of the leucocytes to the damaged endothelium, the cell adhesion molecules (ICAM-1 and VCAM-1) are eliminated by endothelial cells. They are released in the serum and circulate freely as variant structures that have no transmembrane and cytoplasmic divisions.Therefore, they become soluble CAM (sICAM -1 and sVCAM-1). This makes it easy to study them in the peripheral circulation. Some studies find that sVCAM-1 is higher in patients with carotid stenosis compared with healthy controls and has a positive correlation with the plaque instability $(26,32)$.
PEYCHEVA M. et al. Matrix Metalloproteinases (MMP) are a group of proteases involved in matrix degradation and plaque destabilization $(33,34)$. They are a group of zinc-dependent endopeptidases that destroy proteins in the extracellular matrix. MMPs could also influence endothelial cell function and vascular tissue remodeling during various biological processes (35).Some studies try to find connections between the MMP-9 activity and the histological type of the carotid plaque (36). But still it does not mean a reliable serum marker. Some authors prove a connection between the histological features of the plaque and the serum levels of MMP-1, MMP-7and tissue inhibitor of matrix protease (TIMP-1)(33). Recent study finds connection between the serum level of MMP-2 and MMP3 and the ultrasound echogenicity of the plaque (37). Studying the role of MMPs will help not only for finding a good serum marker for plaque destabilization but will help in pharmacotherapy with the use of the MMPs inhibitors.

Fibrinogen plays an important role in the coagulation cascade and its plasma level can easily be measured. Some studies find that fibrinogen is higher in patients with carotid stenosis and even in symptomatic stenosis (38, 39). It can be used as nonspecific indicator of inflammation but not as an independent predictor of atherosclerosis progression (40).

Some pro-inflammatory cytokines are strongly related to the processes of atherosclerosis. IL-6 is associated with presence of carotid plaques and plaque instability $(26,38,41)$. There are some hypothesis that IL-23 and IL-27 are also increased in carotid atherosclerosis $(42,43)$ and IL-18 is connected with plaque destabilization (44). Still their role and action in the inflammation and plaque development is not clear.

\section{CONCLUSION}

Different studies are trying to find reliable biomarkers that can correspond to the mechanism of carotid plaque formation and destabilization. The efforts are directed to find the proper biomarkers that will indicate the presence of atherosclerosis, the degree of the stenosis, plaque instability and the onset of the cerebro-vascular event. It is one step towards stratified and personalized medicine.

\section{ACKNOWLEDGMENTS}

This review is part of the research project "Markers of inflammation associated with the nature and instability of plaques in carotid 
atherosclerosis" funded by Medical University Plovdiv, Bulgaria. (Project code № 04/2015)

\section{REFFERENCES}

1. Esper, R., Nordaby, R., et al., Endothelial dysfunction: A comprehensive appraisal. Cardiovascular Diabetology, 23;5:4, 2006.

2. Hansson, G., Mechanisms of disease: Inflammation, atherosclerosis, and coronary artery disease. New England J. of Medicine, 28;353(4):429-30, 2005.

3. Petrova, J., Manolov, V., Milev, B., et al. Assessment of biomarkers in patients with carotid ath-erosclerosis. Am. J. Neuroprotec. Neuroregen, 7, 72-74, 2015.

4. Tityanova, E., Stamenova, P., et al, National Consensus on Ultrasound Diagnostics and Treatment in Excranial Carotid Pathology, Sofia 2011.

5. Mayerl C., Lukasser M., Sedivy R, et al., Atherosclerosis research from past to present - on the track of two pathologists with opposing views, Carl von Rokitansky and Rudolf Virchow, in Virchows Archiv, 449: 1:96-103, 2006.

6. Hansson G., Libby P., The immune response in atherosclerosis: a double-edged sword. Nature Reviews Immunology, 6:7:508-519, 2006.

7. Kataoka, Y., Nicolls, S. Residual risk and biology of the disease: Implications for plaque imaging. In: Nichols S, Crowe T, eds. Imaging coronary atherosclerosis. New York: Springer.1-21, 2014.

8. Rocha, V., Libby, P. Obesity, inflammation, and atherosclerosis. Nat Rev Cardiol. 6:399-409, 2009.

9. Malek, A., et al. Hemodynamic shear stress and its role in atherosclerosis. JAMA. 282:2035-42, 1999.

10.Widlansky, M., Gokce, N., Keaney, Jr JF, et al. The clinical implications of endothelial dysfunction. J Am Coll Cardiol. 42:1149-60, 2003.

11.Hahn, C., Schwartz, M.. Mechanotransduction in vascular physiology and atherogenesis. Nat. Rev. Mol. Cell Biol. 10:53-62, 2009.

12.Ross R. The pathogenesis of atherosclerosis: a perspective for the 1990s. Nature 362:801-809, 1993.

13.Kwon, G., Schroeder, J., et al.Contribution of macromolecular structure to the retention of low-density lipoprotein at arterial branch points. Circulation 117:2919-2927, 2008.

14.Skagen, K., Skjelland, M., et al.Unstable carotid artery plaque:new insights and controversies in diagnostics and treatment. Croat Med J. 57:311-20, 2016.

15.Moore, K., Tabas, I. Macrophages in the pathogenesis of atherosclerosis. Cell,145:341-355, 2011.

16. Woollard, K. Immunological aspects of atherosclerosis, Clinical Science, 125(5):221-235, 2013

17.Ammirati, E., Moroni, F., Norata, G. Markers of inflammation associated with plaque progression and instability in patients with carotid atherosclerosis. Mediators Inflamm. 2015:718329, 2015.

18.Stoll, G., Bendszus, M. Inflammation and atherosclerosis: novel insights into plaque formation and destabilization. Stroke, 37:1923-1932, 2006.

19.ESC/EAS Guidelines for the management of dyslipidaemia: the Task Force for the management of dyslipidaemias of the European Society of Cardiology (ESC) and the European Atherosclerosis Society (EAS). Eur Heart J, 32:1769-818, 2011.

20.Catapano, A., Pirillo, A., et al. HDL in innate and adaptive immunity. Cardiovascular Research, 103(3):372-383, 2014.

21.Norata, G., Pirillo, A., et al. Emerging role of high density lipoproteins as a player in the immune system. Atherosclerosis, 220(1):11-21, 2012.

22.Ridker, P., Cushman, M., Stampfer, M.. Inflammation, aspirin, and the risk of cardiovascular disease in apparently healthy men. The New England Journal of Medicine. 336(14):973-979, 1997.

23.Ridker, P., Buring, J.,et al. Prospective study of C-reactive protein and the risk of future cardiovascular events among apparently healthy women. Circulation. 98(8):731-733, 1998.

24.Rost, N., Wolf, P., Kase, C.. Plasma concentration of C-reactive protein and risk of ischemic stroke and transient ischemic attack: the Framingham Study. Stroke. 2001;32(11):2575-2579.

25.Puz, P., Lasek-Bal, A., et al. Inflammatory markers in patients with internal carotid artery stenosis. Archives of Medical Science. 9(2):254-260, 2013.

26.Debing, E., Peeters,E.,et al. Markers of inflammation in patients with symptomatic and asymptomatic carotid artery stenosis: a case-control study. Vascular and Endovascular Surgery. 42(2):122-127, 2008.

27.Hashimoto, H., Kitagawa, K., et al. CReactive Protein Is an Independent 
Predictor of the Rate of Increase in Early Carotid Atherosclerosis. Circulation. 104:63-67, 2001.

28.Knoflach, M., Kiechl, S., et al. Pentraxin-3 as a marker of advanced atherosclerosis results from the Bruneck, ARMY and ARFY Studies. PLoS ONE. 7(2), 2012.

29.Savchenko, A., Imamura, M.,et al. Expression of pentraxin 3 (PTX3) in human atherosclerotic lesions. J Pathol. 215(1):4855, 2008.

30.Ley, K., and Huo, Y. VCAM-1 is critical in atherosclerosis. Journal of Clinical Investigation, 107(10), 1209-1210,2001.

31.Chia, M. The role of adhesion molecules in atherosclerosis. Crit Rev Clin Lab Sci. 35(6):573-602,1998.

32.Ragino, Y., Chernjavski ,A.,et al. Oxidation and endothelial dysfunction biomarkers of atherosclerotic plaque instability. Studies of the vascular wall and blood. Bull Exp Biol Med. 153(3):331-335, 2012.

33.Pelisek, J.,et al.Multiple biological predictors for vulnerable carotid lesions. Cerebrovascular Diseases. 28(6):601-610, 2009.

34.Galis, Z., Khatri, J. Matrix metalloproteinases in vascular remodeling and atherogenesis: the good, the bad, and the ugly. Circulation Research. 90(3):251262, 2002.

35.Wang, X., Khalil, R. Matrix Metalloproteinases, Vascular Remodeling, and Vascular Disease. Adv Pharmacol. 81:241-330, 2018.

36.Loftus, I., Naylor, A.,et al. Increased matrix metalloproteinase-9 activity in unstable carotid plaques. A potential role in acute plaque disruption. Stroke. 31(1):40-7, 2000.
PEYCHEVA M. et al.

37.Boukrid, V. Changes of carotid plaque echogenicity over time and serum biomarkers. Abstracts for the 22nd Meeting of the European Society of Neurosonology and Cerebral Hemodynamics (ESNCH). International Journal of Stroke 12(1S):58, 2017.

38.Puz, P., Lasek-Bal, et al. Inflammatory markers in patients with internal carotid artery stenosis. Archives of Medical Science. 9(2):254-260, 2013.

39.Halvorsen, D., Johnsen, S.,et al. The association between inflammatory markers and carotid atherosclerosis is sex dependent: the Tromsø study. Cerebrovascular Diseases. 27(4):392-397, 2009.

40.Sabeti ,S., Exner, M., et al. Prognostic impact of fibrinogen in carotid atherosclerosis: nonspecific indicator of inflammation or independent predictor of disease progression. Stroke. 36(7):1400-4, 2005.

41.Chapman, C., Beilby, J., et al. Monocyte count, but not C-reactive protein or interleukin-6, is an independent risk marker for subclinical carotid atherosclerosis. Stroke. 35(7):1619-1624, 2004.

42.Gregesen, I., Sandanger , Ø., et al. Interleukin 27 is increased in carotid atherosclerosis and promotes NLRP3 inflammasome activation. PLoS One. 27;12(11), 2017.

43.Abbas, A., Gregesen, I., et al. Interleukin 23 levels are increased in carotid atherosclerosis: possible role for the interleukin 23/interleukin 17 axis. Stroke. 46(3):793-9, 2015.

44.Pigarevskii, P., Maltseva, S., et al. Role of interleukin-18 in destabilization of the atherosclerotic plaque in humans. Bull Exp Biol Med. 157(6):821-4, 2014. 